\title{
BEHAVIOR OF NATURAL ORGANISMS AS A MIMICKING TOOL IN ARCHITECTURE
}

\author{
DEENA EL-MAHDY ${ }^{1} \&$ HISHAM S. GABR ${ }^{2}$ \\ ${ }^{1}$ Cairo University, Egypt \& The British Unversity in Egypt, Egypt \\ ${ }^{2}$ Cairo University, Egypt \& American University in Cairo, Egypt
}

\begin{abstract}
The relation in between architecture and nature has been one of combination for the last 400 years. Throughout history, architects have looked to nature for inspirations for building shapes, forms, and ornamentation without understanding nature's behavior. Moreover, new architectural approaches are being called for integrating nature as a tool for solving problems and enhancing adaptation within the context. This has been recently implemented in biomimicry theories that are applied in design processes. Biomimicry is considered a new discipline that studies living organisms' design and behavior in nature to solve human problems. Not only does this help in finding new ways for adaptation, it also generates new sources of inspiration for aesthetic expressions. This is of great importance nowadays as buildings are becoming inefficient; consuming a lot of energy, materials, and resources. Furthermore, construction processes are becoming increasingly unsustainable. While on the other hand organisms are creating effective and intelligent solutions in their homes by using less material. Engineers can also mimic natural methods of construction for building and design rather than their exact shapes. In addition, they also lead to efficiency in terms of energy, material usage, time, effort, and cost and can promote more adaptable, sustainable, and optimum solutions.

This research paper aims to explain the organism's behavior when producing the material and translating it by using a digital tool by mimicking its behavior in construction. Methodology used for this process involves case studies analysis and observations of living organisms' behavior. The cases selected apply biological techniques through their construction process. This involves using natural organisms to produce physical components for architectural product design. Results from the experimental and case studies will aim to conclude a set of findings on approaches for linking organism behavior with future construction processes.

Keywords: biomimicry, computational process, material, organism behavior, performance, rules in nature, responsive, robotic fabrication.
\end{abstract}

\section{INTRODUCTION}

Throughout history, architects and designers have always looked to nature for inspiration. They have not only thought to imitate plants and animals shapes and forms, but they have also tried to find strategies, logic and methods in design that are analogous to nature's process of growth and evolution.

However, architecture and nature share common features as they both have the same logic of growth and adaptation. In the light of this research, most designers translate forms in nature into building materials purely as geometrical forms, without understanding the structure and material logic behind it Oxman [1]. As a result a gap has arisen between methods of physical and digital form-finding that depends on nature, where most designers extract from nature instead of adopting biological systems and learning from them. However, designers shouldn't ignore the relation in between the materials and their shaping processes used in the 
generation of form. In order to fill this gap, new digital technology is changing the way that architects develop their construction process. This is achieved by applying organisms' behavior in the process and studying nature's rules and logic.

\section{RULES AND THEORIES IN NATURE}

As an architect, one should get inspired from nature and learn how organisms adapt with nature to implement their strategies in architectural processes Weinstock [2]. Many rules and systems can be naturally found in plants, animals and humans. Furthermore, different theories are generated from nature such as self-organization, complexity, swarm theory, chaos, fractal, etc., which leads to sustainable and intelligent solutions. The fractal theory for example refers to mathematical ideas, which describe some natural phenomena such as clouds, tornadoes, and snowflakes. These mathematical parameters of fractals could be applied in solving some construction problems. On the other hand, self-organization is a process in which the internal organization of a system adapts to the environment to promote a specific function without being guided or managed from an external source Hensel [3]. In architectural interpretation, self-organization involves local and independent decision making, planning and building without central control.

\section{BIOMIMICRY IN ARCHITECTURE}

In 1997, Benyus defined biomimicry as being from the Greek 'bios', which means life, and 'mimesis', which means imitation. This field studies nature's models and imitates or takes inspiration from its designs and processes to solve human problems. Biomimetic is frequently used in scientific research to explore forms, connections, structures, as well as biological mechanisms and processes. It is not a new concept, but a technical term used in different fields of science. It is also used by material scientists when researching living organisms' properties and their natural systems. This is helpful when recreating these same properties in industrial, medical, and biological products Alquest et al. [4]. The biomimetic inspiration is not about designing and copying forms found in nature, but understanding the behavior of these organisms Yowell [5]. In architecture, it is mostly used in building facades, where the skin is dynamic and it responds to its internal and external environment.

Biomimicry is the study of natural organisms' forms, structures and mechanisms in order to mimic their same functions. It contains two main approaches, the top-down and bottom-up approach, as shown in Figure 1. In the top-down approach, designers look to nature for
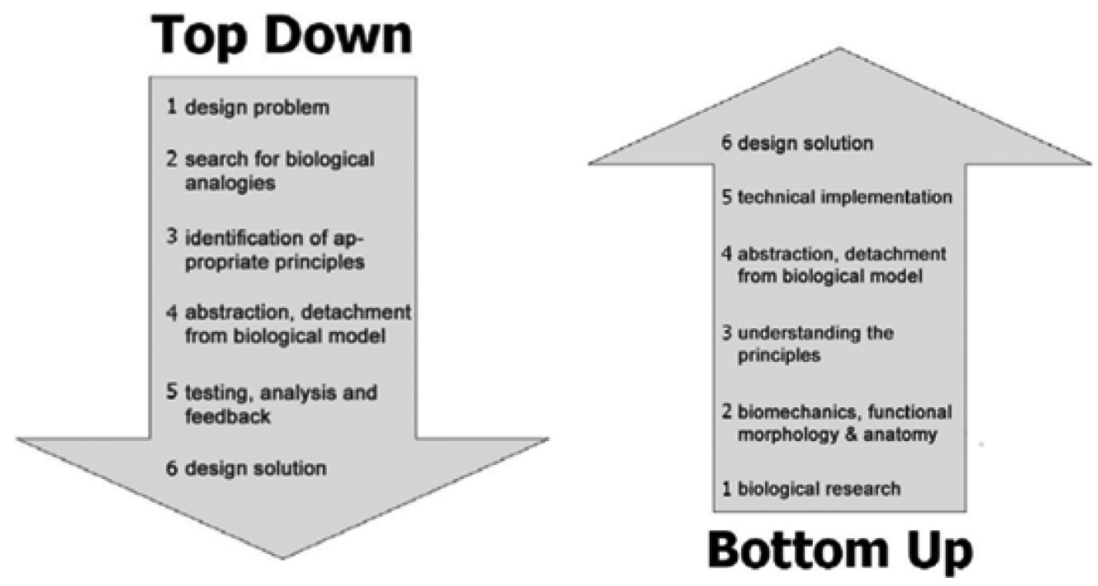

Figure 1: Top-down design and bottom-up approach (Jean Knippers, 2009). 

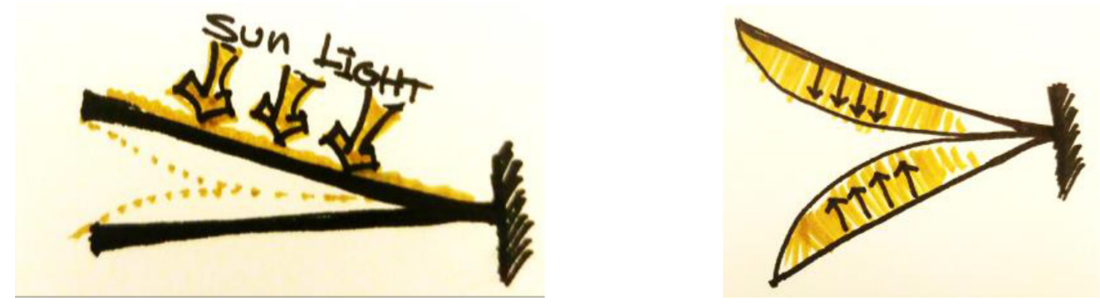

Figure 2: The changes in the material causing by the sun (by Deena El-Mahdy, 2016).

solutions, which may resolve later problems - then biologists match it to organisms that have solved similar issues. This approach is effectively led by designers identifying initial goals and parameters for the design. On the other hand, in the bottom-up approach, the design process is depending on having knowledge of relevant biological or ecological research rather than determined design problems El-Ahmar [6].

The Homeostatic façade, designed by Ned Khan, is an example of the top-down approach. It simulates different types of responses to internal and external conditions in order to control temperature and sunlight. The façade system was inspired by a muscle that produces energy, heat, force and motion, as shown in Figure 2. The façade imitates the human muscles: when the unit gets heated by the sun, it rapidly opens up, causing the surface to extend. The building skin gradually closes to protect the indoor environment from the outer environmental condition. The intelligence of the smart material allows the building to adapt to its ecological conditions.

\section{BEHAVIOR OF NATURAL ORGANISMS' APPROACH}

In the natural world, each organism has its own different characteristics. These characteristics appear through their behavior, structure, form, pattern, and material. This differs not only from one species to another, but also within the same species itself. The two forthcoming illustrations discussed in this section display the magnificent role of the spiders' and bees' behavior in designing their homes, producing their material and their ability to save materials in building their colonies and structures. It also displays their efficiency in terms of energy, material usage, time, effort, and cost. Their behavior can promote more adaptable, sustainable, and optimum solutions. Solutions extracted from these organisms could be applied in solving our architectural and structural problems through the building process.

\subsection{The structure of spider's silk and its behavior}

Spider's silk is one of the engineering miracles and it is considered to be one of the strongest materials compared to its scale. The secret of the silk lies in its fiber atoms. Its molecular structure determines silk elasticity and simultaneously makes it extremely resistant to rupture by mechanical force. Silk fiber is ten times stronger than Kevlar, five times tougher than steel and twice as elastic as nylon. The silk fibers emerge from spinnerets, as each spinneret has spigots that produce a liquid material that twist and connect together to produce the silk and give it its strength, as shown in the Figure 3, ACMilanillo [7].

On the other hand, spiders use silk for many purposes; reproduction, protection and hunting. Moreover, the web is made of composite silks; the joint and the spindle knot droplet. The genius of the web can be seen in the water droplets in each junction (knots) that allow the web to flex and stretch without causing any damage, as shown in Figure 4. 

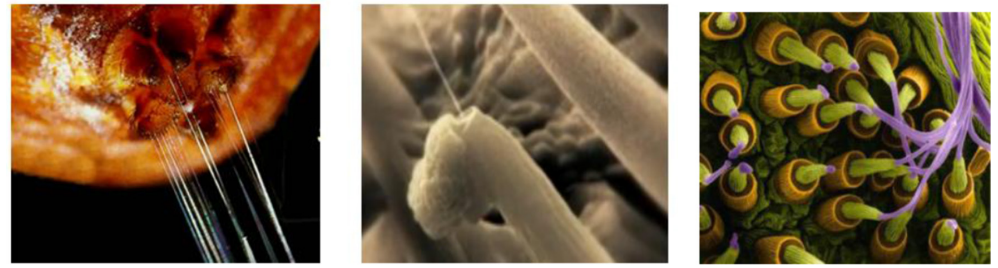

Figure 3: Spiders' spinnerets that produced the liquid fibers (left pic. by Jim Stephens, 2015; right pic by Dennis Kunkel, 2004).
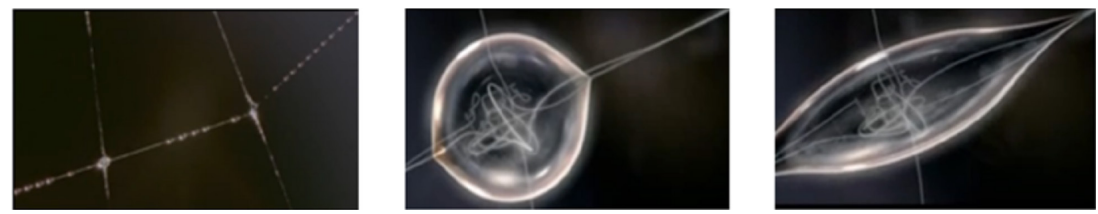

Figure 4: The junction that giving the web its flexibility (by youtube video, BBC Invisible World spider the genius architect, ACMilanillo, 2015).

Silks produced by the same spiders can have different features. Moreover, there are five different types which could be produced by the same spider with special properties, variation strength, extendibility and behavior. In the light of this research, architects nowadays get inspired from nature and learn some intelligent and efficient solutions from this process. Furthermore, by applying this process of spiders; the robotic industry could use special digital techniques which could save a lot of material to reach the optimum solution in less time.

\subsection{The structure of the honeycomb and the bees' behavior}

Another illustration could be observed in the morphology of the honeycomb structure. The honeycomb structure is considered to be one of the most magical structures in nature Menges [8]. The genius of the beehive lies in its hexagonal structure and the material used, as shown in Figure 5. Different techniques could be mimicked in order to reach the most efficient solutions with fewer resources.

In the light of this research, the use of the hexagonal structure allows minimal effort for the bees with fewer bee-waxes. By looking inside the hive shape, as shown in Figure 5, it is apparent that the units are connected side by side. The hive not only provides the bees with a place to stay, but also to store their nectar until it turns to honey. Space efficiency can be observed in their wax unit, which fits the honey produced from one bee. The wax needs a lot of effort to be produced, as $8 \mathrm{oz}$. of honey is equal to $1 \mathrm{oz}$. of bee-wax. This reflects the genius behavior of the bees in producing their material and calculating the space needed to save a lot of material which could be used as an alternative for constructing different solutions. As shown in Figure 6, the gaps appear if the honeycomb was circular or octagonal and the area would shrink if it takes a triangular or quadrangle structure.

This led to the most efficient shape; the hexagonal form. This shape allows storing the largest possible amount of honey to build the most spacious living space by using a minimum amount of wax. The bees' method in finding the optimum and most efficient solution could be implemented digitally in architectural fabricating techniques. 

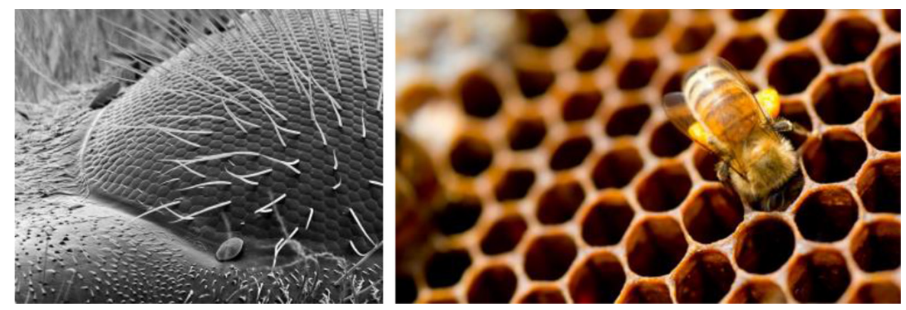

Figure 5: The hexagonal structure of the eye bees on the left (by Rose-Lynn Fisher, 2013, the honeycomb by Katrice Wilson, 2014).
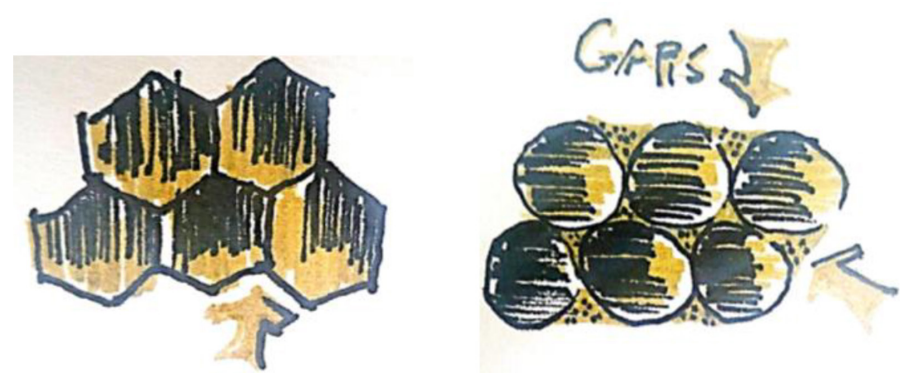

Figure 6: The differentiation between the shapes (by Deena El-Mahdy, 2016).

\section{METHODOLOGY OF COMPUTATION AND DIGITAL FABRICATION}

Considerable case studies have been proposed in the light of this research which deals in such a way that it can also observes the living organism behavior. The specific cases discussed in this research apply biological techniques through their construction process inspired from organism's behavior. This involves using natural organisms to produce physical com ponents for architectural product design. Results from these cases will conclude a set of findings on approaches for linking organisms' behavior with future construction processes. These cases can demonstrate how architectural and biological levels can be adapted with the environment.

\subsection{Case study 1: Silkworm pavilion - MIT Lab: Neri Oxman}

At Silk pavilion, Neri Oxman and her research group at MIT followed nature's strategy. In this project, the pavilion was designed and constructed in two phases: digitally fabricating a scaffolding envelope made of silk fibers and installing thousands of silkworms to spin a secondary silk envelope. A set of apertures built into the initial envelope capture's the light and the heat, thus controlling the distribution of silkworms on the structure.

\subsubsection{Inspiration and design concept}

This pavilion demonstrates the architectural potential of a building method inspired by natural organisms such as the silkworm's behavior and its ability to generate a 3D cocoon out of a single multi-property silk thread (1 km in length). The design process occurs through controlling the environment in which the silkworm grows Zolotovsky [9]. 

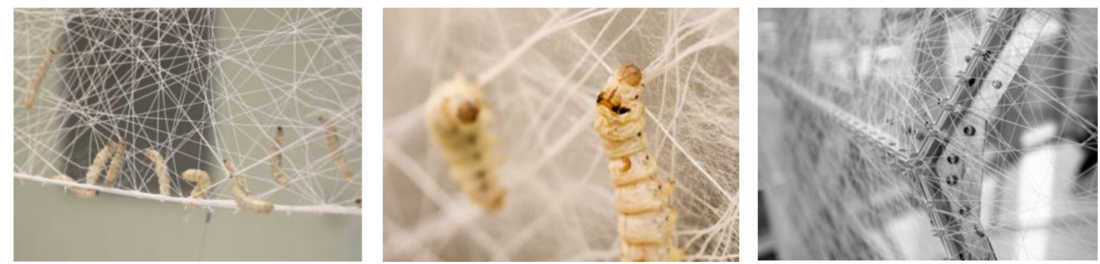

Figure 7: Silkworms spins silk fiber while fabrication process (matter media MIT, 2013).
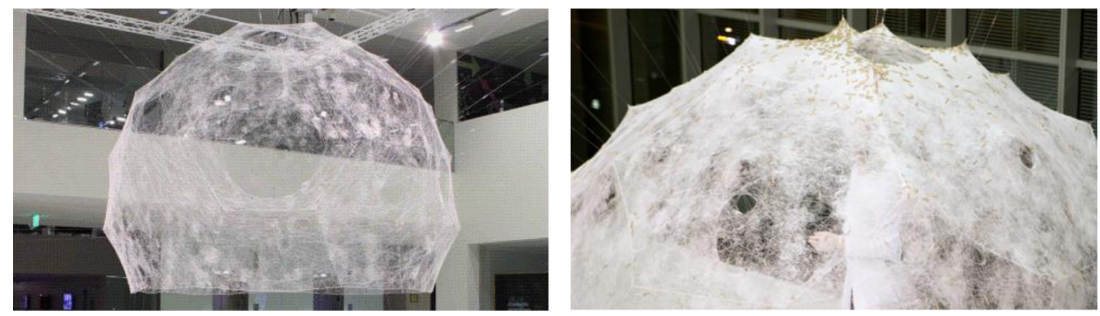

Figure 8: Silkworms spin fibrous around the pavilion (matter media MIT, 2013).

\subsubsection{The pavilion process (Silkworms' behavior)}

The silkworm was the main designer that spins the silk on the pavilion. Around 26 polygonal silk panels based on silk worm cocoons are constructed by a CNC machine. Then they cover the structure by silkworms and let them sew, as shown in Figure 7. The worms automatically spun silk to reinforce the weak points present within the structure areas, as shown in Figure 8. The material that was used in this pavilion was the silk of the worms.

\subsubsection{Digital fabrication}

The digital approach was developed to support the findings with regard to the worm's possible range of motion and deposition behavior, thus enabling digital fabrication tools and biological construction to merge Oxman et al. [10].

\subsubsection{Computational design}

The geometry of the pavilion was created by using an algorithm that assigns a single continuous thread across patches providing various degrees of density, functional density gradients formed by environmental constraints such as light and heat Oxman et al. [11].

\subsubsection{Summary}

Regardless to the direct potential for biological fabrication combined with digital fabrication, the experimental data shows that the relationship between scaffold surface morphology and biological fibre organization can be considered valuable Oxman et al. [10]. It is evident that silkworms' behavior could control our design, material and process to reach an efficient solution in our architectural and construction processes. This leads to the construction of buildings that are highly efficient, more durable, and sustainable that requires fewer resources such as energy, material and time.

\subsection{Case study 2: ICD/ITKE research pavilion: Achim Menges}

At ICD pavilion, Achim Menges and his research group at ICD followed nature's strategy. In this project, the pavilion was designed and constructed digitally. Through a novel robotic 
fabrication process an initially flexible framework was gradually stiffened by reinforcing it with carbon fibres from the inside. The resulting lightweight fibre composite shell formed the pavilion with unique architectural qualities, which consists of highly efficient-material structure. These prototypes explore application potentials for novel computational design, simulation and fabrication processes in architecture Menges [12].

\subsubsection{Inspiration and design concept}

This pavilion shows the architectural potential of a building method inspired by the underwater nest construction of the water spider (Agyroneda Aquatica), as shown in Figure 9.

The design concept is based on the study of biological construction processes for fiber-reinforced structures. These processes are relevant for applications in architecture, as they do not require complex framework. Thus, the web building and construction process of the spiders was examined and the underlying behavioral patterns and design rules were analyzed, abstracted and transferred into a technological fabrication process. This example illustrates how efficient reinforced structures could be created from adaptive fabrication techniques.

\subsubsection{The pavilion process (Water spider's behavior)}

In order to mimic the biological formation sequences in building construction methods, a process was devised where an industrial robot was placed in air supported by a membrane envelope. This envelope is supported by air pressure and is reinforced from the inside with carbon fiber by the help of robots Menges [12].

\subsubsection{Digital robotic fabrication}

Consistent to the adaptive computational design strategy, a prototype robotic fabrication process was developed to reinforce the fibers within the pavilion. The robotic current position and force is recorded via an embedded sensor to adapt to these parameters during the production process system.

\subsubsection{Computational design}

The cyber-physical system, as shown in Figure 10, documents the process that occurs between production and the digital generation of robot control. After mimicking the spider's behavior, they replace this motion by a robotic arm that constructs the fibers through the pavilion. This provides new opportunities for adaptive robotic construction processes, as shown in Figure 11 Menges [12].
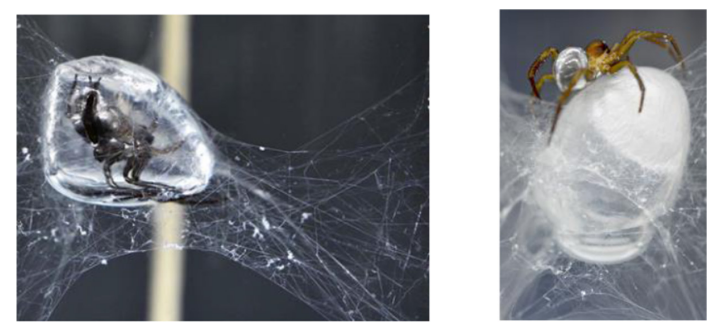

Figure 9: Diving Bell Water Spider reinforcing an air bubble from the inside (ICD/ITKE University of Stuttgart, 2014). 


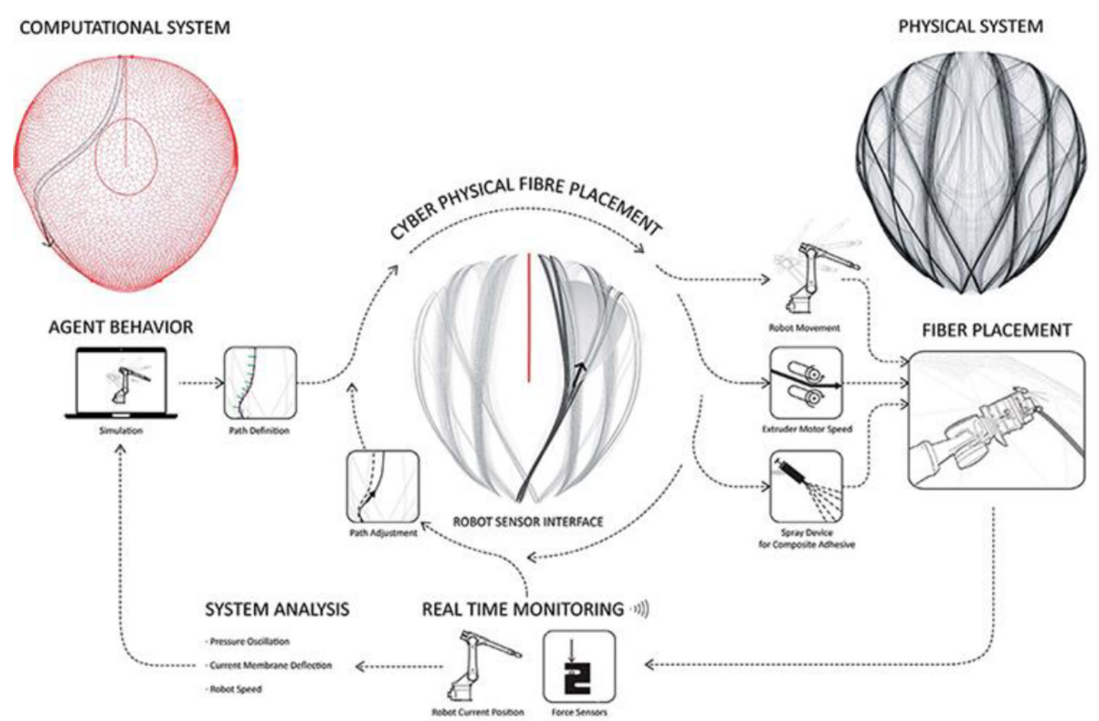

Figure 10: Cyber-physical fiber placement process, conceptual fabrication (ICD/ITKE University of Stuttgart, 2014).
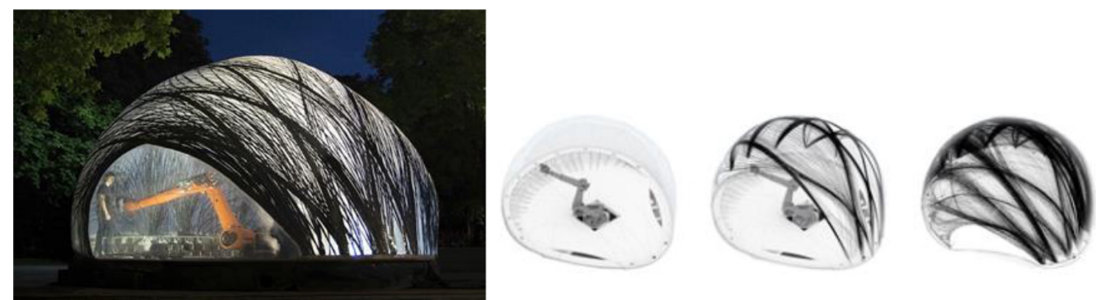

Figure 11: Robotic fiber reinforcement (ICD/ITKE University of Stuttgart, 2014).

\subsubsection{Summary}

Regardless of the direct potential for biological fabrication combined with digital fabrication, the experimental data shows that there is a relationship in between the spider's behavior through reinforcing its home similarly by reinforcing biological fiber in the pavilion. It is evident that spiders' behavior could control our design, material and process to reach an efficient solution in our construction processes. The result is not only an effective-material construction, but also an innovative and expressive architectural demonstrator.

\section{EXPERIMENTAL CASE STUDY}

In this project, the research group formed under the supervision of MagLab followed the nature's strategy. The experimental installation was designed and constructed in two phases: manually fabricating the units and the units' mechanism as a responsive façade by mimicking spiders' movement.

\subsection{Inspiration and design concept}

The unit's form a shape inspired by the spiders' mechanism, the units are connected together by using wires that are attached to Arduino in order to control the movements. This installation 
was proposed to be a part of a façade. When the sun heats the unit in the middle, it acts in such a way that it closes the openings of other units. These units, blocks the direct sun light from entering the space, as shown in Figure 12. The material that is used for this process is semitransparent so that it can allow the sun light to enter the space.

\subsection{The pavilion process fabrication}

In order to mimic the biological formation of the spider in this installation, units were fabricated and connected together in such a way that once the middle unit opens, it grabs the other units around which produces the motion.

Consistent to the adaptive computational design strategy, a prototype fabrication system process was developed to control the units' movement inside the façade installation. The installation's current position is connected with wires together in such a way that it joins the embedded Arduino sensor to adapt these parameters during the transmission of light, as shown in Figure 13.
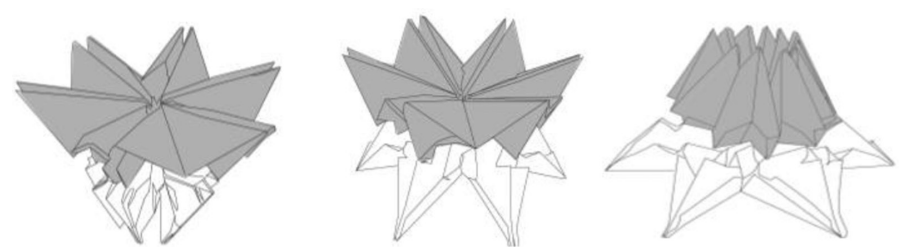

Figure 12: The mechanism of the units (by Deena El-Mahdy, 2012).
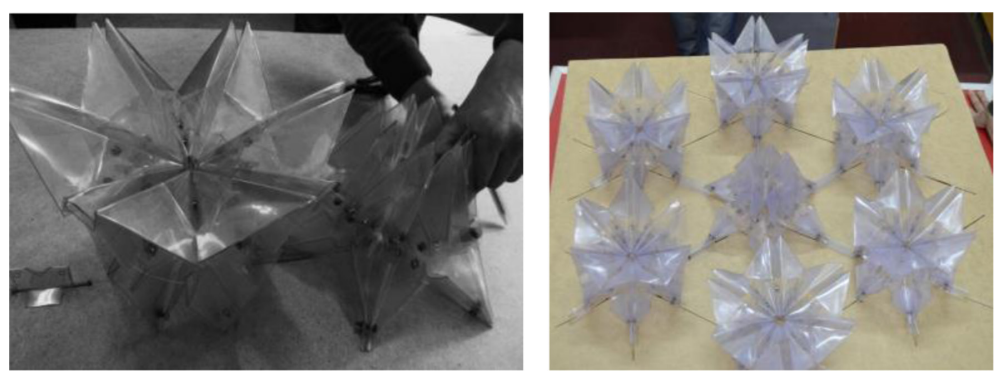

Figure 13: The mechanism of the units connected by wires attached to Arduino (by Deena El-Mahdy, 2012).
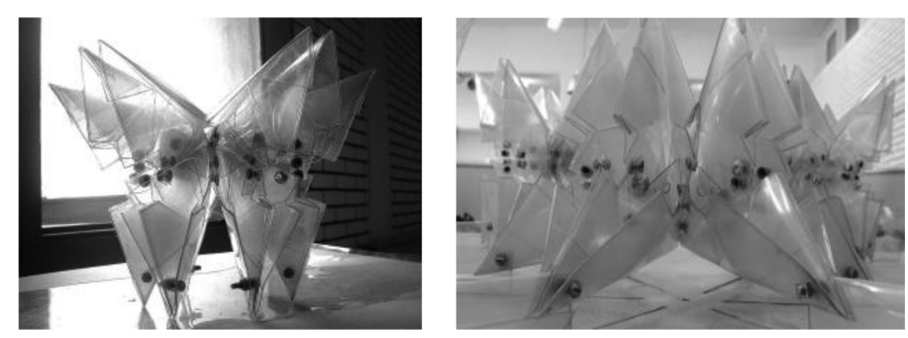

Figure 14: The units connected by wires and attached to Arduino (by Deena El-Mahdy, 2012). 


\subsection{Summary}

Regardless to the direct potential for biological fabrication combined with digital mechanism, the experimental installation shows us the understanding of the organisms. How it enlightens their behaving strategy by fabricating the entire process manually, which could be an alternative for solving the design problems. It is evident that organisms behave in such a way that it controls the design and reaches an efficient solution for constructing different processes.

\section{CONCLUSION}

Concerning the impact of organisms' behavior on the design process, this research paper has analyzed some of these parameters in implementing them for the architectural and construction disciplines to reach an optimum solution.

Two case studies were presented to observe the organism's behavior. The results presented in this research illustrate the possibility of mimicking organism's behavior and their material performance for the construction process of the two pavilions. The biomimicry approach shows how these problems can be solved by revisiting and by observing the nature's and organisms' performance.

The integration of both fields allows architects the possibility of collaborating between different fields of science. In addition, architecture and engineering have developed computational tools for evaluating and simulating complex physical performances. It is evident that these parameters could control the proposed design, material and can reach efficient solutions for constructing processes.

To conclude, the revolution of computational design allows designers to integrate natural design parameters into construction design process. It is apparent that fabrication is redefined today as an integral part of the design process. Indeed, fabrication is not only a prototyping technique, but also a change in the generation, which leads to methods that are highly efficient, more durable and sustainable.

\section{REFERENCES}

[1] Oxman, N., Material Based Design Computational, MIT, PhD. thesis, Ch. 4, Massachusetts Institute of Technology, pp. 99-120, 2010.

[2] Weinstock, M., Metabolism and morphology. Architectural Design: Versatility and Vicissitude, 78(2), p.33, 2008.

[3] Hensel, M., Computing self-organization: environmentally sensitive growth modelling. Architectural Design, 76(2), p.13, 2006.

[4] Alquest, S., Kampowski, T., Torghabehi, O.O., Menges, A. \& Speck, T., Development of a digital framework for the computation of complex material and morphological behavior of biological and technological systems. Computer Aided Design, 60, pp. 84-104, 2015. http://dx.doi.org/10.1016/j.cad.2014.01.013

[5] Yowell, J., Biomimetic Building Skin: A Phenomenological Approach Using Tree Bark As Model, MSc. thesis, University of Oklahoma, pp. 17-23, 2011.

[6] El-Ahmar, S., Biomimicry as a Tool for Sustainable Architectural Design, MSc. thesis, Ch.1, Alexandria University: Egypt, p. 10, 2011.

[7] ACMilanillo, youtube video, BBC Invisible World spider the genius architect, 2015.

[8] Menges, A., Computational morphogenesis integral form generation and materialization processes. Proceedings of the 3rd International ASCAAD Conference on Em'body'ing Virtual Architecture, Alexandria: Egypt, p. 735, 2007. 
[9] Zolotovsky, K., Bio Constructs - Method for Bio-Inspired and Bio-Fabricated Design, MSc. Thesis, Ch.1, Massachusetts Institute of Technology, p. 12, 2012.

[10] Oxman, N., Laucks, J., Kayser, M., Duro, J. \& Uribe, C., Silk pavilion: a case study in fibre-based digital fabrication. FABRICATE Conference Proceedings, Negotiating Design \& Making, eds F. Gramazio, M. Kohler, S.L. Enber, ta Verla, p. 254, 2014.

[11] Oxman, N., Laucks, J., Kayser, M., Duro, J. \& Uribe, C., A biologically-informed finite element approach to structural performance and material optimization of robotically deposited fibre structures. eCAADe 31 Conference on Computation and Performance, eds R. Stouffs \& S. Sariyildiz, ecaade, Delft University, 2, pp.1-10, 2013.

[12] Menges, A., ICD/ITKE Research Pavilion, 2014-15, available at www.icd.unistuttgart.de/. 